\title{
Radiometric block adjustment of hyperspectral image blocks in the Brazilian environment
}

\section{Gabriela Takahashi Miyoshi, Nilton Nobuhiro Imai, Antonio Maria Garcia Tommaselli, Eija Honkavaara, Roope Näsi \& Érika Akemi Saito Moriya}

To cite this article: Gabriela Takahashi Miyoshi, Nilton Nobuhiro Imai, Antonio Maria Garcia Tommaselli, Eija Honkavaara, Roope Näsi \& Érika Akemi Saito Moriya (2018) Radiometric block adjustment of hyperspectral image blocks in the Brazilian environment, International Journal of Remote Sensing, 39:15-16, 4910-4930, DOI: 10.1080/01431161.2018.1425570

To link to this article: https://doi.org/10.1080/01431161.2018.1425570

册Published online: 16 Jan 2018.

Submit your article to this journal

LII Article views: 294

View Crossmark data $₫$

Citing articles: 5 View citing articles 주 


\title{
Radiometric block adjustment of hyperspectral image blocks in the Brazilian environment
}

\author{
Gabriela Takahashi Miyoshia, Nilton Nobuhiro Imaia,b, \\ Antonio Maria Garcia Tommasellia,b, Eija Honkavaarac, Roope Näsic \\ and Érika Akemi Saito Moriya ${ }^{b}$
}

\begin{abstract}
aPostgraduate Program in Cartographic Science, School of Technology and Science, São Paulo State University (UNESP), Presidente Prudente, Brazil; ${ }^{b}$ Department of Cartography, São Paulo State University (UNESP), Presidente Prudente, Brazil; 'Department of Remote Sensing and Photogrammetry, Finnish Geospatial Research Institute FGI, Masala, Finland
\end{abstract}

\begin{abstract}
The objective of this investigation was to study and optimize a hyperspectral unmanned aerial vehicle (UAV)-based remotesensing system for the Brazilian environment. Comprised mainly of forest and sugarcane, the study area was located in the western region of the State of São Paulo. A novel hyperspectral camera based on a tunable Fabry-Pérot interferometer was mounted aboard a UAV due to its flexibility and capability to acquire data with a high temporal and spatial resolution. Five approaches designed to produce mosaics of hyperspectral images, which represent the hemispherical directional reflectance factor of targets in the Brazilian environment, are presented and evaluated. The method considers the irradiance variation during image acquisition and the effects of the bidirectional reflectance distribution function. The main goal was achieved by comparing the spectral responses of radiometric reference targets acquired with a spectroradiometer in the field with those produced by the five different approaches. The best results were achieved by correcting the bidirectional reflectance distribution function effects and by applying a least squares method to a radiometric block adjustment using only the image data, thereby achieving a root mean square error of $11.35 \%$.
\end{abstract}

\section{ARTICLE HISTORY}

Received 26 June 2017

Accepted 27 December 2017

\section{Introduction}

The use of unmanned aerial vehicles (UAVs) as remote-sensing platforms has increased explosively in recent years (Colomina and Molina 2014). These types of platforms are highlighted by their scalability, persistence, flexibility, technologies, and opportunities, as well as their ability to fly in dangerous environments (Everaerts 2009). They can be used in many application areas, such as archaeological, agricultural, and forestry assessment and monitoring. UAVs have lower operational costs than airborne platform-based systems and are feasible for the acquisition of higher temporal resolution data than 
conventional manned airborne platforms. Moreover, UAVs can fly below clouds, thus offering a weather-resistant remote-sensing tool. The rapid development of miniaturized hyper- and multispectral-sensor technologies has also enabled the capture of high spatial and spectral resolution images with UAVs, which opens up totally new remotesensing opportunities (Saari et al. 2013; Aasen et al. 2015).

In several recent studies, pushbroom scanning-based hyperspectral sensors have been implemented in UAVs (Zarco-Tejada, González-Dugo, and Berni 2012; Hruska et al. 2012; Büttner and Röser 2014; Suomalainen et al. 2014; Lucieer et al. 2014). Miniaturized hyperspectral imagers operating in a 2D frame format principle are a novel sensing approach for the capture of spectral signatures (Mäkynen et al. 2011; Saari et al. 2013; Honkavaara et al. 2013; Näsi et al. 2015; Aasen et al. 2015). A 2D frame format provides strong geometric and radiometric constraints due to its rigid rectangular geometry and multiple overlapping images (Honkavaara et al. 2012a). This framework offers interesting possibilities for UAV remote sensing because it results in more stable imaging geometries than pushbroom scanning and uses fewer ground control points (GCPs) and lower grade inertial navigation systems (INSs).

For remote-sensing applications, e.g. forest, hydrological, and agricultural monitoring, radiometric calibration is a fundamental requirement since the rigorous analysis of the spectral features of targets is commonly employed to extract information. Therefore, radiometric calibration must minimize and correct sensor-related nonuniformities, effects of illumination changes caused by cloud coverage, and effects of the bidirectional reflectance distribution function (BRDF) (Honkavaara et al. 2009). Several recent studies have used reflectance targets and the empirical line method to perform reflectance transformations (Laliberte et al. 2011; Aasen et al. 2015). Another popular approach is to utilize physical radiative transfer modelling-based correction approaches utilizing either in situ atmospheric observations or default atmospheric parameters (Richter and Schläpfer 2002; Zarco-Tejada, González-Dugo, and Berni 2012; Büttner and Röser 2014). For multiview imaging technologies that capture image blocks with overlapping frame images, radiometric block adjustment-based approaches are highly relevant. They are based upon observations of the same object in different images from different directions. The method assumes that the object should provide a similar reflectance in all images, and thus, a model is developed to simulate the differences in radiometric values in different images (Honkavaara et al. 2012b, Honkavaara et al. 2013). Hakala et al. (2013) integrated an additional irradiance sensor attached to a UAV to compensate for illumination variations into this method. This method has been used previously in the context of agricultural applications (Honkavaara et al. 2013; Hakala et al. 2013) and in forest applications (Näsi et al. 2015).

The objective of this investigation was to develop and assess a UAV-based approach for capturing hyperspectral image mosaics in a Brazilian environment constituted of tropical forest and sugarcane plantation areas. The steps to achieve this goal included (1) defining the spectral setting of the camera; (2) applying a radiometric correction and spectral band matching for the images; (3) performing a radiometric block adjustment; (4) checking the best parameters for the radiometric block adjustment based on quality control information; and (5) analysing and concluding information about the most suitable technique considering the regional characteristics of illumination. 


\subsection{Study area}

Located in the western region of São Paulo State $\left(22^{\circ} 23^{\prime} 53^{\prime \prime} \mathrm{S}, 52^{\circ} 31^{\prime} 00^{\prime \prime} \mathrm{W}\right)$, the study area comprises a protected area managed by the Chico Mendes Institute of Biodiversity Conservation (ICM-Bio) that was created by the Brazilian Government to conserve and manage natural resources. The area belongs to the Ponte Branca fragment of the Black Lion Tamarin Federal Reserve and covers an approximate area of 11 ha that is mainly classified as forest within a sugarcane plantation neighbourhood. According to the Köppen classification system, the regional climate is tropical wet with dry seasons in the winter (June-September) and intense summer rains (December-March).

In the beginning of the summer (December), cloud cover is denser due to the summer rains that can occur at any hour of the day. After the summer and during the autumn, there are still clouds during the day, but they were less intense than those during the summer. During this time, the velocity of the wind increases, causing a variation in the cloudiness. The minimum cloud cover occurs during the middle of the winter (August) through the dry season and increases in the spring. The capability to operate in cloudy conditions is thus important for a remote-sensing system due to the high probability of cloud cover.

The area of surveying was almost flat and was divided by a road leading to a forest towards the south and to a sugarcane field towards the north. The sugarcane field was more than $2 \mathrm{~m}$ high during the date of the aerial survey. The sugarcane was roughly uniform since it possessed areas infected with the mosaic virus and red stripe disease, which degrade this type of crop. Almost the entire cultivated area was densely covered by sugarcane, making the influence of the background insignificant. However, in some regions where the coverage density was lower, the influence of soil on the spectral response was greater. The forested area was at the initial stage of regeneration due to the proximity of the road and the sugarcane plantation. It was mainly composed of pioneer species such as Eugenia uniflora and Dilodendron bipinnatum. Moreover, there were different levels of height, with trees up to $15 \mathrm{~m}$ above the ground.

\section{Materials and methods}

\subsection{UAV and hyperspectral camera}

The platform used for the acquisition of the hyperspectral images was developed by Sensormap Geotechnology, a UAV octocopter, model SX8 (Figure 1(a)). The UAV is controlled by the DJl Wookong-M autopilot with flight time of $30 \mathrm{~min}$ with a $5 \mathrm{~kg}$ payload. A set of sensors was attached to the UAV platform, consisting of a frame format hyperspectral camera based on a Fabry-Pérot interferometer (FPI) with one irradiance sensor and one global positioning system (GPS) receiver, an INS Novatel SPAN-IGM S1, and a Raspberry $\mathrm{Pi} \mathrm{B}+$, which was used to record the raw INS data, which was further post-processed to provide the position and attitude data of the platform.

The FPI camera (model DT-0014a) belonging to São Paulo State University (UNESP) used in this work is a commercial camera that was constructed in 2014 by Senop Ltd. (Figure 1(b)). With an adjustable air gap between the FPI, different spectral bands can be acquired in the range of $500-900 \mathrm{~nm}$ with a minimum spectral resolution of $10 \mathrm{~nm}$ at 
(a)

(b)

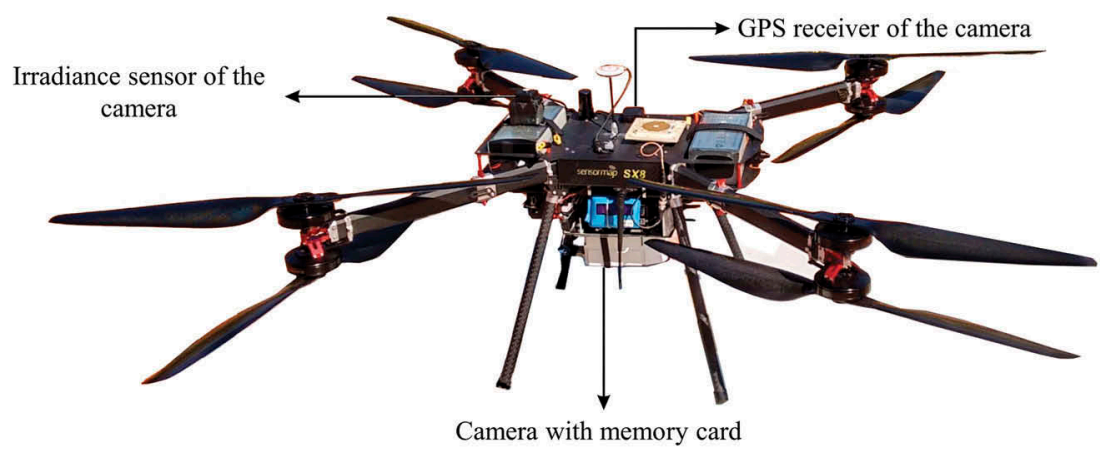

Camera with memory card

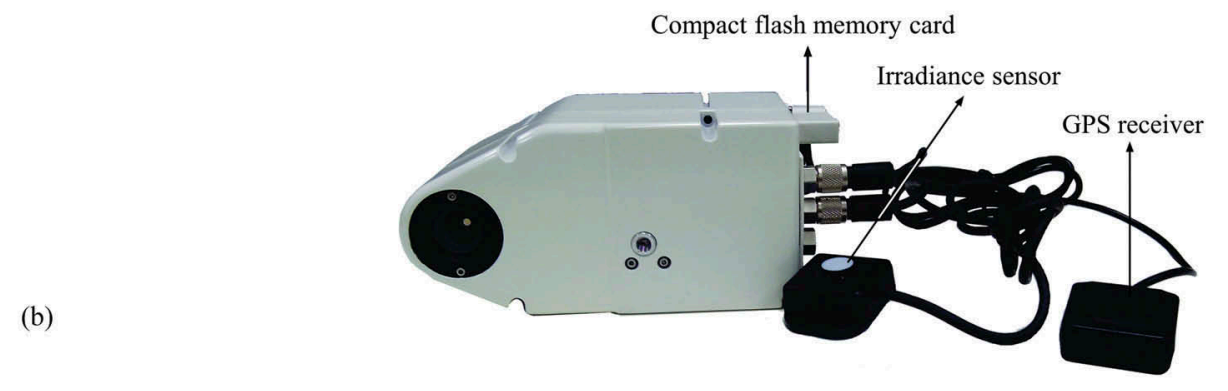

Figure 1. (a) The SX8 UAV, and (b) a hyperspectral camera based on a piezoactuated FPI with a GPS receiver, an irradiance sensor, and a compact flash memory card.

the full width at half maximum (FWHM). The camera system is described briefly below, and details of the camera are given by Oliveira, Tommaselli, and Honkavaara (2016).

The UNESP FPI camera has two CMOSIS CMV400 complementary metal oxide semiconductor sensors to acquire up to two spectral bands simultaneously. The custom optics of the camera comprises an uncalibrated focal length of $8.6 \mathrm{~mm}$, an $f$-number of 2.8 and a field of view (FOV) of $37^{\circ}$ in the image $x$ and $y$ directions. The dimensions of the frame sensor are $1023 \times 1023$ pixels with a square pixel size of $5.5 \mu \mathrm{m}$. In practical applications, when operating as a standalone device, images of the data cube have $1023 \times 648$ pixels in the no-binned mode using a maximum of 25 spectral bands, which are selected flexibly for each application. In addition, it is possible to select the exposure time in consideration of the application and weather conditions during fieldwork.

The camera also has its accessories which are a GPS receiver and an irradiance sensor, both are connected to the camera. The single frequency GPS receiver records the position (latitude, longitude) and the GPS time of the first spectral band of each image. The positioning data recorded by this sensor were not used because the payload of the UAV system has an INS integrated to it, with a dual frequency Global Navigation Satellite System (GNSS) receiver and an inertial measurement unit (IMU) that provides a more accurate position and orientation for each image. To correlate the position and orientation provided by the INS, the time information of each image was used.

The irradiance sensor has a photodetector with cosine collector optics and is used to measure the incident irradiance at the instant of each image acquisition in the spectral 
sensitivity range of $400-1000 \mathrm{~nm}$. However, the sensor is not calibrated to measure in physical units of $\left[\mathrm{W} \mathrm{sr}{ }^{-1}\right]$. During the flight, images with respective position and irradiance data are recorded in a compact flash memory card. The total weight of the system, including the camera and accessories, is lower than $720 \mathrm{~g}$ with dimensions of $77 \mathrm{~mm} \times 89.5 \mathrm{~mm} \times 172.7 \mathrm{~mm}$ (Senop 2017), which are feasible for use in UAV platforms.

The major complication of the camera is the sequential spectral band acquisition. The need to change the air gaps of the FPI as well as the presence of two frame sensors and platform movement produces a hyperspectral data cube in which spectral bands are not perfectly aligned, resulting in a slightly different position between bands of the same image. This band alignment issue is resolved in the image post-processing phase.

\subsection{Determining the spectral setting of the camera}

The hyperspectral setting of the camera was optimized for this application in consideration of the spectral responses of vegetation in the study area. Leaf samples from eight vegetation species were collected in the field and were stored in Styrofoam boxes to preserve their spectral properties until their measurement in the laboratory. The species were (1) Pouteria ramiflora, (2) Croton floribundus, (3) Astronium graveolens, (4) Aspidosperma ramiflorum, (5) Handroanthus avellanedae, (6) Hymenaea courbaril, (7) Eugenia uniflora, and (8) Sugarcane.

Vegetation spectra were acquired in the laboratory using a portable spectroradiometer, a FieldSpec ${ }^{\circledR}$ Handheld UV/NIR from Analytical Spectral Devices (ASD). The acquisition was performed following the geometry suggested by McCoy (2005) to avoid burning the leaves with the halogen lamp as well as to prevent shadows from the instrument and the influence of neighbouring spectral responses. One leaf of each species was measured (Figure 2) because the purpose of this task was mainly to define the spectral configuration of the camera, not to determine the spectral signatures of the samples. Moreover, the predefined central wavelength and FWHM for each air gap of the FPI were used, resulting in non-integer values for the spectral bands. Table 1 shows the 25 spectral bands selected.

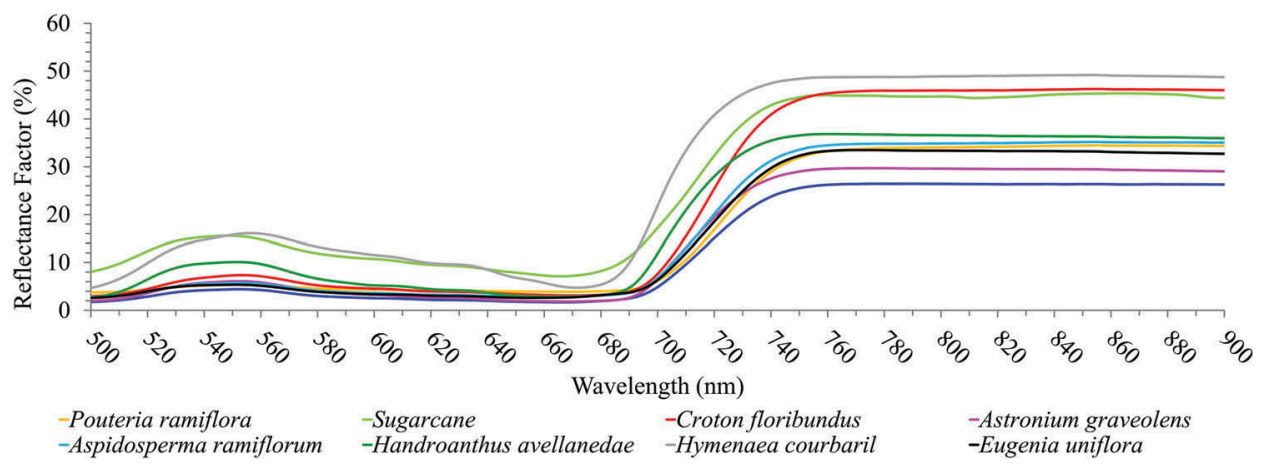

Figure 2. Reflectance factor of species belonging to the study area used to define the spectral bands of the FPI camera. 
Table 1. Spectral settings for the hyperspectral camera $(\lambda=$ central wavelength of the spectral band).

\begin{tabular}{cccccccccc}
\hline$\lambda(\mathrm{nm})$ & FWHM (nm) & $\lambda(\mathrm{nm})$ & FWHM (nm) & $\lambda(\mathrm{nm})$ & FWHM (nm) & $\lambda(\mathrm{nm})$ & FWHM (nm) & $\lambda(\mathrm{nm})$ & FWHM $(\mathrm{nm})$ \\
\hline 506.07 & 15.65 & 580.08 & 15.14 & 650.28 & 15.85 & 699.62 & 21.89 & 749.65 & 19.43 \\
520.00 & 17.51 & 591.49 & 14.67 & 660.27 & 24.11 & 709.71 & 20.78 & 770.46 & 19.39 \\
535.45 & 16.41 & 605.64 & 13.82 & 669.96 & 21.70 & 719.99 & 20.76 & 790.21 & 18.50 \\
550.76 & 15.16 & 619.55 & 14.59 & 680.06 & 21.00 & 729.56 & 21.44 & 810.15 & 17.66 \\
\hline
\end{tabular}

\subsection{Field work and flight}

The image acquisition was carried out on 19 May 2015, in the beginning of the dry season. The UAV was operated by Sensormap Geotechnology. A flying altitude of $160 \mathrm{~m}$ above the terrain was maintained with a flight speed of $4 \mathrm{~m} \mathrm{~s}^{-1}$, providing images with a ground sample distance (GSD) of approximately $10 \mathrm{~cm}$. The exposure time was $10 \mathrm{~ms}$. The image block contained two image strips with a forward overlap exceeding $50 \%$ and a side overlap greater than $30 \%$. Each flight strip was $850 \mathrm{~m}$ long and $100 \mathrm{~m}$ wide. The aerial surveying was carried out between 12:55 and 13:09 (UTC-3) when the Sun zenithal and azimuthal angles varied from $42^{\circ} 46^{\prime} 12^{\prime \prime}$ to $43^{\circ} 27^{\prime} 36^{\prime \prime}$ and from $349^{\circ} 54^{\prime} 36^{\prime \prime}$ to $345^{\circ}$ $13^{\prime} 12^{\prime \prime}$. During the aerial surveying, the illumination conditions varied due to the different levels of cloud cover.

Three radiometric reference targets made with ethyl vinyl acetate (EVA) in white, grey, and black with nominal reflectances of $85 \%, 47 \%$, and $4 \%$, respectively, were distributed in the area. The targets had dimensions of $0.8 \mathrm{~m} \times 0.5 \mathrm{~m}$. They were used to calculate the absolute parameters used to correlate the digital number (DN) of the images with radiometric data collected in the field. The ASD spectroradiometer, using the geometry described by McCoy (2005) and Milton et al. (2009), was used to collect data in the field to determine the hemispherical conical reflectance factor (HCRF) (Schaepman-Strub et al. 2006) for each reference target.

Additional radiometric reference targets were distributed in the area to control the accuracy of the image processing. Seven radiometric reference targets made with EVA were used. In addition, natural targets were used to check the radiometric processing. These targets were composed of both healthy and diseased sugarcane leaves (infected with the mosaic virus), canopies of weed, and sandy soil. Figure 3 shows the spatial distribution of the targets and pictures of the targets in the field.

The radiometric targets were measured in the field using the ASD spectroradiometer just after the imaging acquisition. The measurements were taken using a one degree FOV with a nadir view $1 \mathrm{~m}$ above the target. However, the sugarcane leaves were measured at $30 \mathrm{~cm}$ above the leaves to acquire a more representative spectra about the degree of health of the sugarcane.

First, the ASD spectroradiometer was optimized using a Spectralon ${ }^{\circledR}$ plate to determine the integration time and to correct the dark current. Second, a series of ten measurements was acquired to minimize random errors that may occur during data acquisition. Then, the average spectra for each target was calculated. Finally, to acquire the final spectra of the targets, a mean smoothing filter of seven points was applied to minimize random noise (Tsai and Philpot 1998).

The irradiance sensor of the camera was attached to the top of the UAV (Figure 1(a)). This sensor collected relative irradiance data for each image to provide information 
(a)

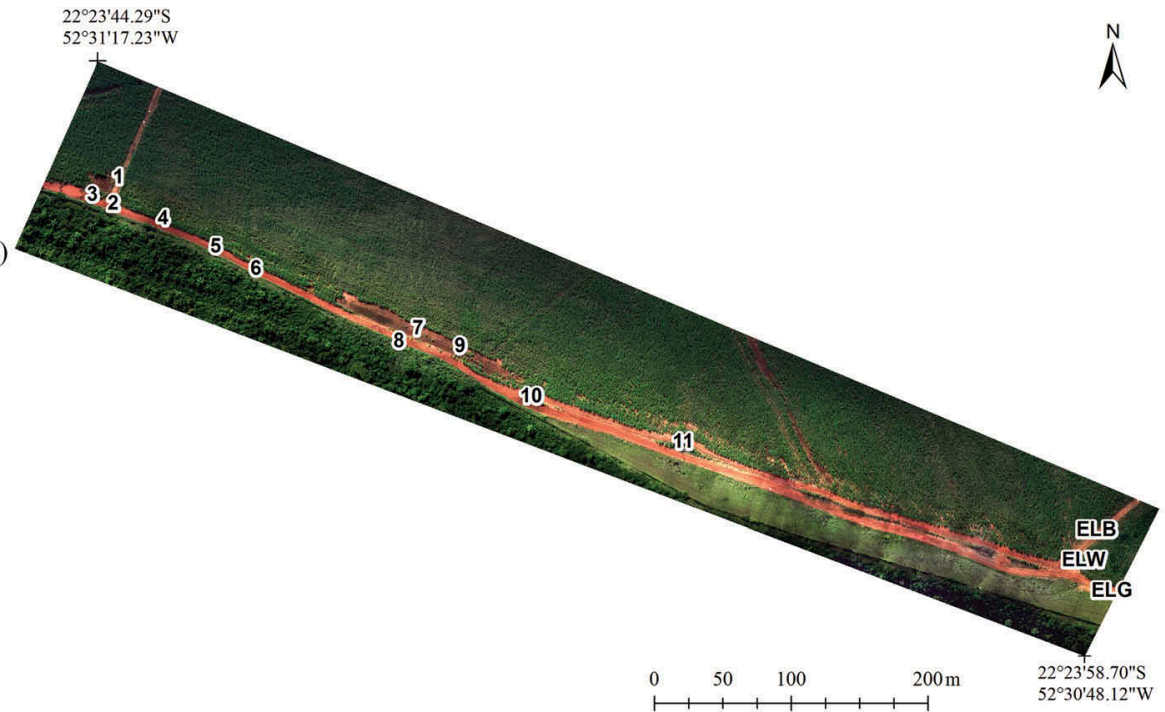

(b)

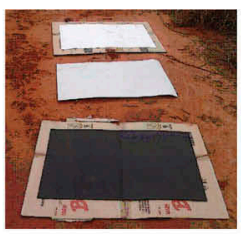

(c)

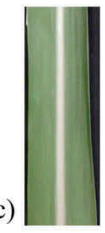

(d)

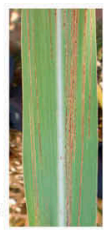

(e)

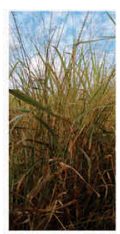

(f)

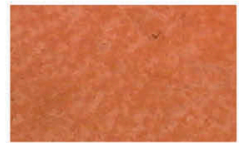

Figure 3. Spatial distribution of the radiometric reference targets, which are used for the imaging processing and quality control tasks. (a) Study area; (b) EVA in white, grey, and black (targets 1, 2, 5, $8,9,10,11$, ELG - grey target used for the empirical line method with a mean HCRF of 47\%, ELW white target used for the empirical line method with a mean HCRF of $85 \%$, and ELB - black target used for the empirical line method with a mean HCRF of 4\%); (c) Healthy sugarcane (target 3); (d) diseased sugarcane (target 4); (e) weed (target 7); and (f) sandy soil (target 6).

about the incident radiant flux in the imaged area. Irradiance data were also acquired in the field using the ASD spectroradiometer to support the image processing tasks.

The ASD spectroradiometer was also placed in the study area to acquire irradiance data. It was located in the western part of the study area at the beginning of the flight area and was situated close to target 3 (Figure 3(a)). The spectroradiometer collected radiance data with a Spectralon ${ }^{\circledR}$ plate using a one degree FOV. The irradiance was then calculated based on Rees (2013). In sequence, the irradiance data from the ground were associated with each image by using their acquisition times. Furthermore, spectral resampling (Stein, van der Meer, and Gorte 2006), as illustrated by Equation (1), was applied to determine a single irradiance measurement for each image comprising the spectral range of the camera,

$$
E_{i, j}=\int\left(E(\lambda) R_{j}(\lambda) d \lambda\right) / \int\left(R_{j}(\lambda) d \lambda\right)
$$

where $E$ is the spectral irradiance measurement of the image $i$ in the spectral range of the camera for band $j ; E(\lambda)$ is the irradiance measurement for each wavelength $\lambda$ in the 
desired spectral range; and $R_{j}$ is the spectral response function of each spectral band. In the case of the FPI camera, the spectral response function of each band was approximated using a Gaussian function.

\subsection{Hyperspectral imaging processing}

Images acquired with an FPI hyperspectral camera are processed similarly to regular frame format imagery. However, specific processing steps are required due to the timesequential operating principle of the camera (Honkavaara et al. 2013). Figure 4 presents the workflow adopted to process the FPI images.

Aiming to transform the DN into radiance values with units of [photon pixel ${ }^{-1} \mathrm{~s}^{-1}$ ] and to correct noise caused by dark current, radiometric calibration parameters provided by Senop Ltd. and the dark imagery acquired before the flight are required. The software Hyperspectral Imager is used, provided by Senop Ltd., to apply dark current and radiometric corrections and transform the binary raw images into the Environment for Visualizing Images image format file (*.dat), a flat-binary raster file with an American Standard Code for Information Interchange header file as complement.

A band registration is required because of the time-sequential operating principle of the camera, which causes misalignments of the individual bands of the hypercubes. This camera requires $0.90 \mathrm{~s}$ to acquire 25 spectral bands using an integration time of $10 \mathrm{~ms}$. Considering the flight height and speed of the UAV used to image acquisition, $160 \mathrm{~m}$ and $4 \mathrm{~m} \mathrm{~s}^{-1}$, respectively, the distance between corresponding pixels in the first and in the last spectral band was around 32 pixels, in the flight line direction. The average difference between corresponding pixels in two sequential spectral bands is less than 2 pixels (1.33 pixels). To correct this displacement, we used the software provided by Senop Ltd. for the band registration. It uses features of interest and 2D transformations to align the bands. Tommaselli et al. (2015) also show an assessment of spectral bands registration from images acquired with this camera.

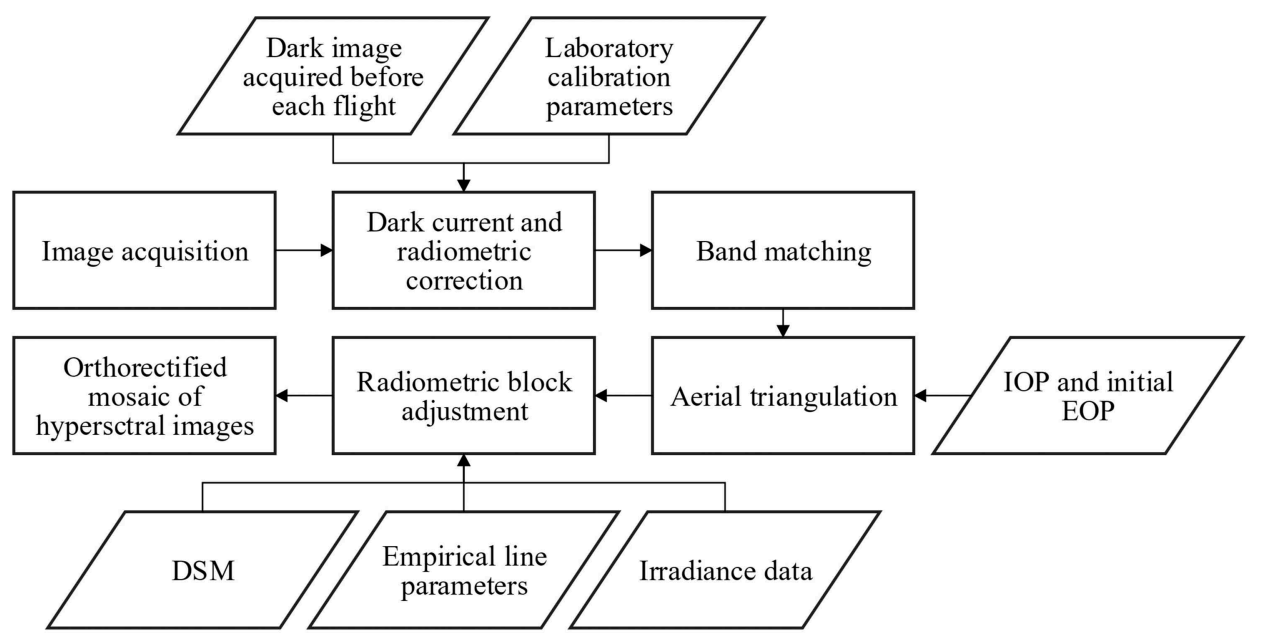

Figure 4. Workflow used to process the image acquired with the FPI hyperspectral camera. 
Aerial triangulation was carried out to calculate accurate exterior orientation parameters (EOP) for each image. A band centred on $709.71 \mathrm{~nm}$ was used in the aerial triangulation; it was empirically chosen as a reference since it showed good contrast between the objects in the scene. The photogrammetry module of Erdas Imagine was used to perform the aerial triangulation and is used with the pre-calibrated interior orientation parameters from Berveglieri and Tommaselli (2016). The EOP measured with a Novatel SPAN S1 INS and post-processed in Waypoint Inertial Explorer software were used as weighted constraints in the bundle block adjustment, with a standard deviation of $50 \mathrm{~cm}$ for the perspective centres positions and $10^{\circ}$ for the attitude angles. In addition, five GCPs located in the corners and middle of the area were used to perform the bundle block adjustment, in which the initial values of the EOPs, provided by the INS, were refined using tie points and GCPs.

The BRDF effects and illumination variations were corrected by applying a radiometric block adjustment approach as proposed, and developed, by Honkavaara et al. (2012a, 2012b, 2013) and Hakala et al. (2013). In this study, different parameters were applied for the radiometric processing to verify the set that best fits the Brazilian environment. The method used in this study is described in Section 2.5.

Finally, an orthorectified hyperspectral reflectance mosaic was calculated by applying the geometric and radiometric model parameters. For geometric processing, a digital surface model (DSM), which was calculated using images acquired from a previous flight and supplied by the Topocart Company, was used. Berveglieri et al. (2016) reveal more details about the image processing procedure used to calculate the DSM with a $45 \mathrm{~cm}$ GSD, which was resampled to $10 \mathrm{~cm}$ in this work.

\subsection{Radiometric block adjustment}

A radiometric block adjustment is based on the assumption that the same object should provide a similar DN in all of the images in which it appears (Honkavaara et al. 2012b, Honkavaara et al. 2013). The method uses the grey DN values of the radiometric tie points in the overlapping images as observations, and it determines the model parameters describing the differences among the DNs in the different images (i.e. the radiometric model) indirectly via the weighted least squares principle. In this study, we used the average reflectance in an object area of $2 \mathrm{~m} \times 2 \mathrm{~m}$ for the radiometric tie points. The model for the DN is given in Equation (2),

$$
\mathrm{DN}_{\mathrm{j}_{, \mathrm{k}}}=a_{r e l_{-j} j}\left(a_{a b s} R_{j, k}\left(\theta_{i}, \theta_{r}, \varphi\right)+b_{a b s}\right)
$$

where $R_{j, k}\left(\theta_{\mathrm{i}}, \theta_{\mathrm{r}}, \varphi\right)$ is the bidirectional reflectance factor of the object point $k$ in an image $j ; \theta_{i}$ and $\theta_{r}$ are the respective illumination and reflected light (observation) zenith angles, $\varphi_{\mathrm{i}}$ and $\varphi_{\mathrm{r}}$ are the azimuth angles, respectively, and $\varphi=\varphi_{\mathrm{r}}-\varphi_{\mathrm{i}}$ is the relative azimuth angle; $a_{\mathrm{abs}}$ and $b_{\mathrm{abs}}$ are the parameters for the empirical line model for transforming the reflectance into DN values (Smith and Milton 1999); and $a_{\text {rel } \_j}$ is the relative correction parameter with respect to the reference image to correct for the illumination differences between the images. The linear BRDF model of Walthall et al. (1985) was implemented by Honkavaara et al. (2013) to model the effects of anisotropy. The parameters used can be selected according to the demands of the data set in consideration. 
Table 2. Corrections applied in the radiometric bundle adjustment.

\begin{tabular}{|c|c|c|}
\hline Correction method & $\begin{array}{l}\text { BRDF } \\
\text { correction }\end{array}$ & $a_{\mathrm{rel} / j}$ \\
\hline No correction (no_corr) & No & 1 (constant) \\
\hline Only BRDF correction (BRDF_only) & Yes & 1 (constant) \\
\hline $\begin{array}{l}\text { BRDF correction and relative parameters calculated from } \\
\text { the irradiance sensor of the camera (BRDF_rela_cam) }\end{array}$ & Yes & $\begin{array}{l}\text { Initial value from the radiance sensor of the } \\
\text { camera. Refined based on image } \\
\text { information }\end{array}$ \\
\hline $\begin{array}{l}\text { BRDF correction and relative parameters calculated from } \\
\text { the ground (BRDF_rela_ground) }\end{array}$ & Yes & $\begin{array}{l}\text { Initial value from the irradiance sensor on the } \\
\text { ground. Refined based on image } \\
\text { information }\end{array}$ \\
\hline $\begin{array}{l}\text { BRDF correction and initial relative parameters with the } \\
\text { same value (BRDF_rela) }\end{array}$ & Yes & $\begin{array}{l}\text { Initial value of } 1 \text {. Refined based only on image } \\
\text { information }\end{array}$ \\
\hline
\end{tabular}

In this study, various approaches to calculate the value of $a_{\text {rel_j }}$ were tested. Hakala et al. (2013) proposed a multiplicative correction factor $\left(a_{\text {rel }_{-} j}\right)$ based on irradiance measurements acquired during image acquisition. This correction factor, shown in Equation (3), is calculated for each image by dividing the irradiance of the reference image by the irradiance of the other images,

$$
a_{r e l} j=E_{r e f} / E_{j}
$$

where $E_{j}$ and $E_{\text {ref }}$ are the spectral irradiance measurements during the acquisition of an image $j$ and a reference image ref. In the case of UAV measurements, the non-calibrated values were used. For the ground spectrometer, the relative correction factor was calculated for each band. The relative correction factors were treated as observations in the adjustments, and the a priori standard deviations for the purposes of weighting were set to 0.05 .

Five different correction approaches were applied to the dataset. First, no corrections were applied (no_corr). Next, the BRDF correction was applied (BRDF_only), but a constant value was used for the multiplicative correction factor $\left(a_{\text {rel }} j=1\right)$. Two methods based on in situ irradiance observations were applied to calculate the value of $a_{\text {rel_j}}$ : the irradiance data from the FPI camera's sensor (BRDF_rela_cam) and the irradiance data from the field (BRDF_rela_ground). Finally, in the last approach, a constant value was used as the initial value in the radiometric block adjustment $\left(a_{\text {rel_ } j}=1\right)$, after which this parameter was adjusted using only image information (BRDF_rela). These image processing options are summarized in Table 2.

\subsection{Accuracy assessment}

The accuracy assessment of the hyperspectral image mosaics included an evaluation of the internal uniformity and the external accuracy. The internal uniformity of the mosaics was evaluated using a coefficient of variation (CV) over the block area (Honkavaara et al. $2012 b$ ). It was derived by calculating the CV of each radiometric tie point and then calculating the average CV value for all of the tie points.

In situ reflectance measurements were used to carry out an absolute accuracy assessment of the mosaic. This included seven radiometric reference targets made with EVA, which were not used to calculate the empirical line parameters, and the four natural targets (see details in Section 2.4). The HCRF values of the field 
measurements were compared to the hemispherical directional reflectance factor (HDRF) values obtained from the mosaics, and the root mean square error (RMSE) was calculated to evaluate the best radiometric correction.

First, the in situ measurements acquired using the ASD spectroradiometer were applied to spectral resampling (see Equation (1) in Section 2.3) to calculate the HCRF of each spectral band of the camera. Then, since the coordinates of each target were known, they were located within the orthorectified hyperspectral mosaic, and the values of the HDRF were obtained for the pixel corresponding to those coordinates.

\section{Results and discussion}

\subsection{Irradiance data}

Figure 5 presents the relative irradiance measurements of each image that were acquired both by the irradiance sensor of the camera attached atop the UAV and by the ASD spectroradiometer on the ground. At the beginning of the flight, during the first flight line towards the southeast, the observed irradiance measurements from the ground and the UAV sensors were consistently similar, demonstrating a rising irradiance level (0.60-1.20 $\mathrm{W} \mathrm{sr}^{-1}$ on the ground). After the UAV turned around, the UAV-mounted sensor measured a very low irradiance level, whereas the ground-based irradiance remained quite constantly within the range from 1.00 to $1.20 \mathrm{~W} \mathrm{sr}^{-1}$. The variations in the beginning of the flight were caused by illumination variations induced by clouds; during the second flight line, the low irradiance level in the UAV was likely due to the tilting of the irradiance sensor away from the Sun. Also, the $800 \mathrm{~m}$ distance between the UAV and field station might have caused some differences in the irradiance levels measured on the ground and by the UAV.

\subsection{Radiometric block adjustment}

The aforementioned processing scheme provided different sets of values for the $a_{\text {rel_ } j}$ parameters (Figure 6) for each correction, image, and spectral band. By analysing Figure 6 , it is possible to verify that for BRDF_rela_cam, the relative parameters tried

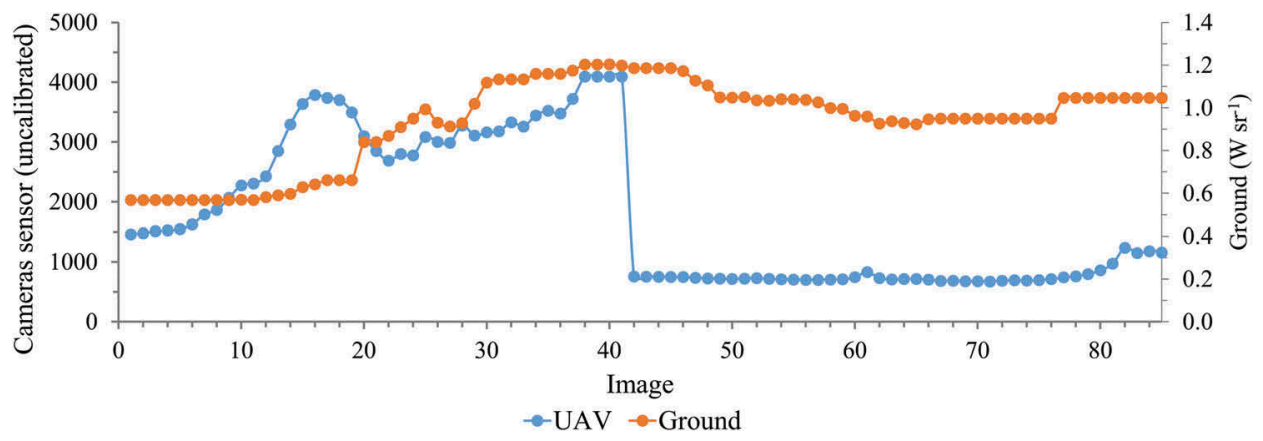

Figure 5. Relative irradiance measurements acquired by the irradiance sensor of the hyperspectral camera attached to the UAV (left, blue line) and by the spectroradiometer on the ground (right, red line). 
(a)

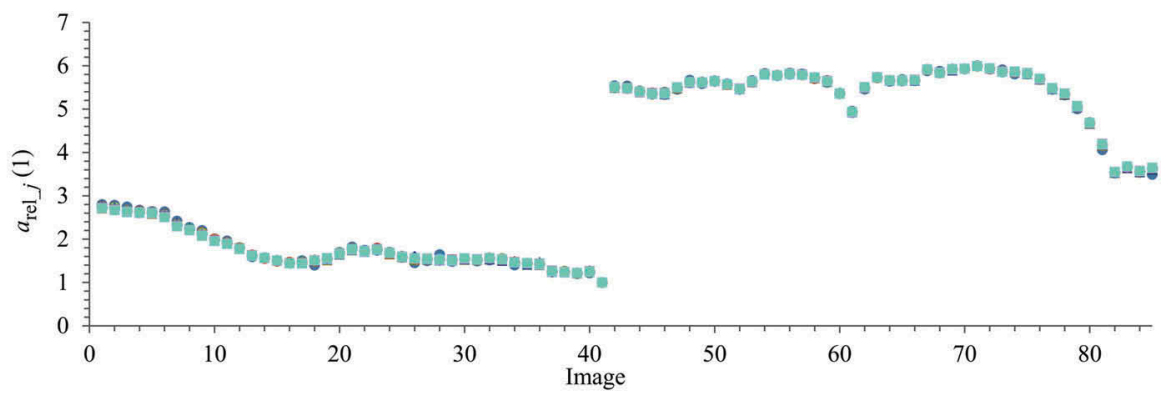

(b)

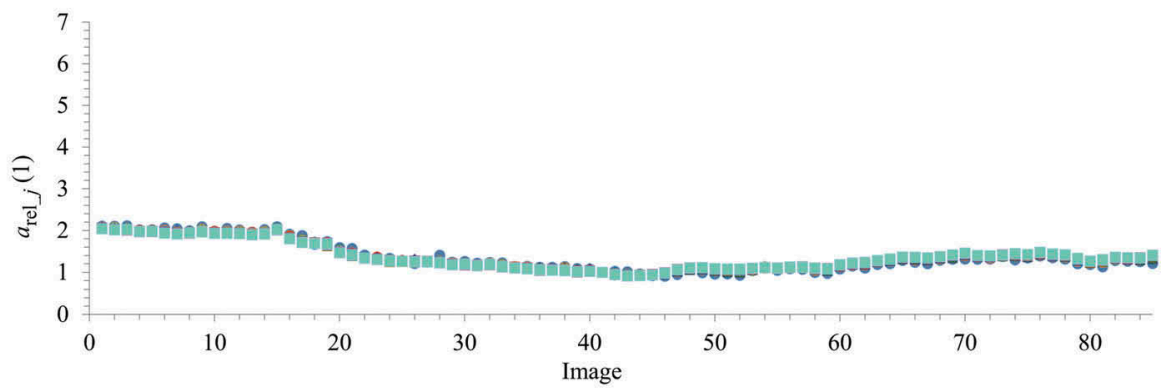

(c)

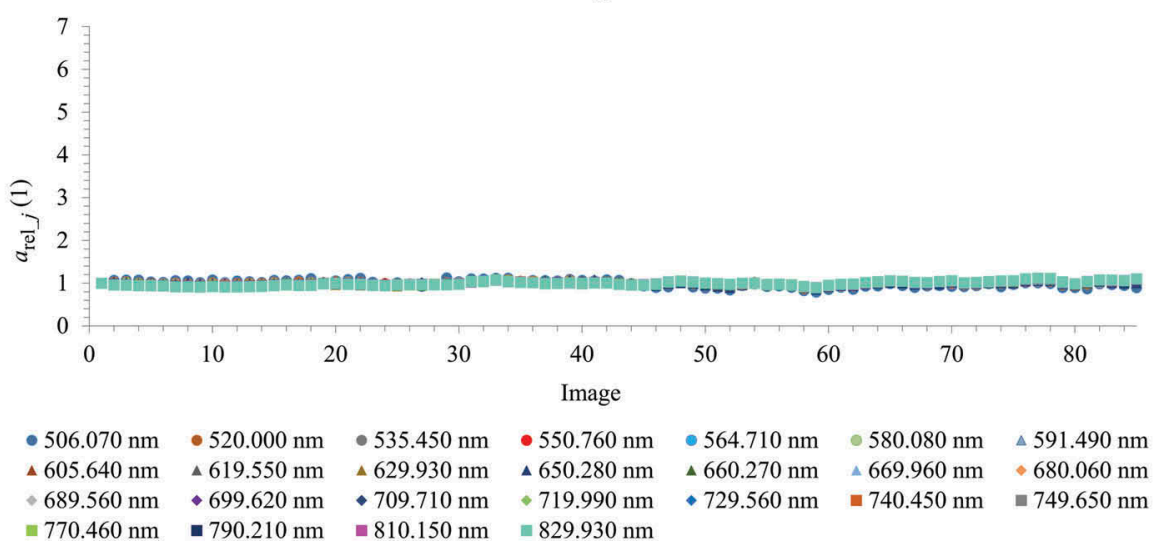

Figure 6. Final relative parameters $\left(a_{\text {rel_j}} j\right)$ for the corrections for each image and spectral band: (a) BRDF_rela_cam, (b) BRDF_rela_ground, and (c) BRDF_rela.

to compensate for higher values of the irradiance that were registered by the UAV sensor in the first flight strip. In the second flight strip, the irradiance values were lower, and consequently, the relative parameters were higher. The behaviour of the relative parameters in BRDF_rela_ground was similar, although they demonstrated a lower variance of $a_{\text {rel_j}}$, which could be explained by the lower variance of the irradiance data acquired by the ground spectroradiometer compared with the data from the UAV.

Moreover, it is observed that for all of the images in BRDF_rela_cam and for most of the images in BRDF_rela_ground, the values of $a_{\text {rel_j}}$ were higher than one, suggesting the irradiance flux was lower for the other images than for the reference image, which is confirmed by Figure 5. When the parameters of BRDF_rela are considered alone, it is observed that the irradiance flux in all of the images are almost the same because the values of $a_{\text {rel_j }}$ are nearly one. 
(a)

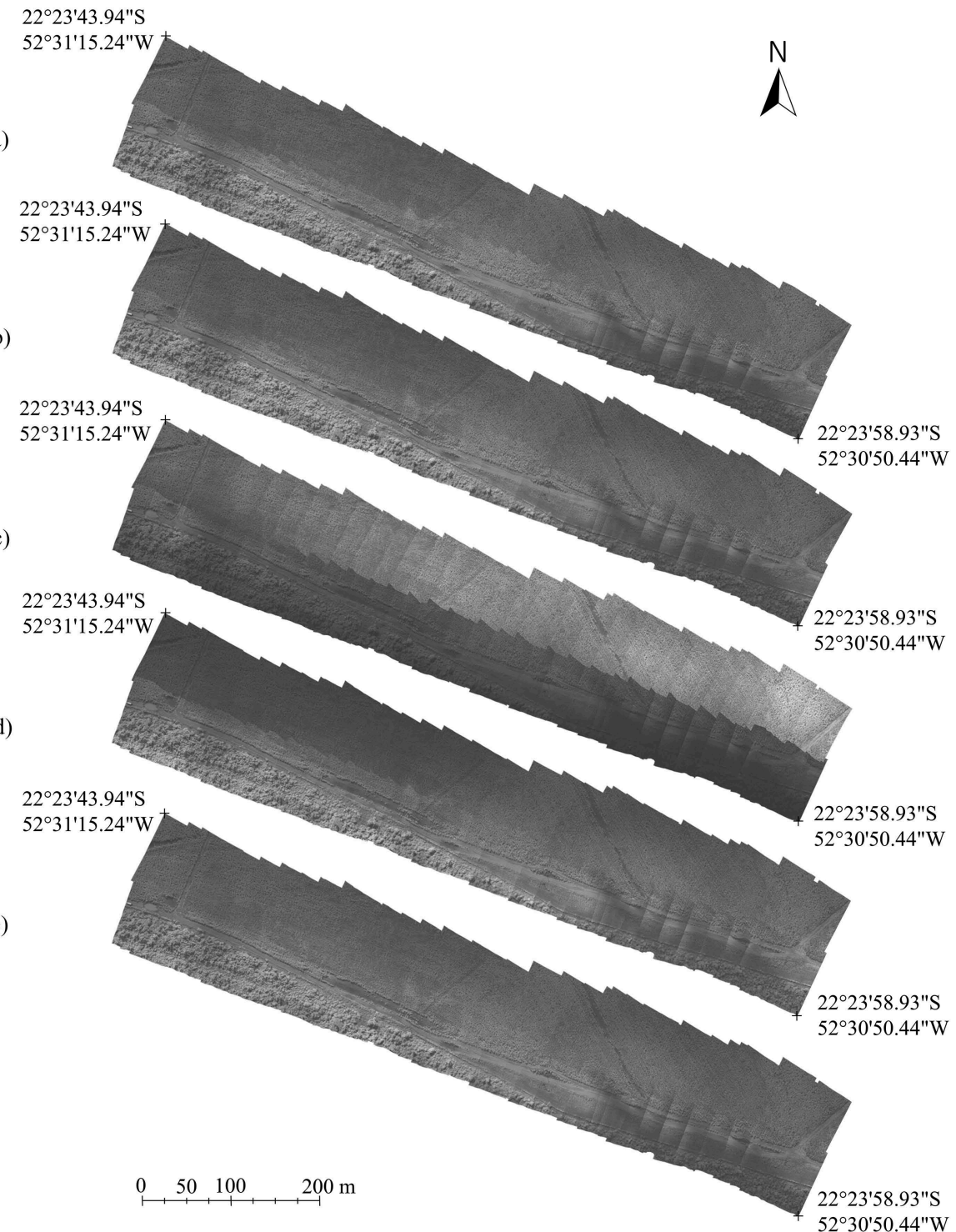

Figure 7. Enhanced mosaics (band centred on $749.65 \mathrm{~nm}$ ) for the five different parameters applied during the radiometric bundle block adjustment: (a) no_corr; (b) BRDF_only; (c) BRDF_rela_cam; (d) BRDF_rela_ground; and (e) BRDF_rela.

Using different parameters for the radiometric block adjustment, five different hyperspectral mosaics were produced (Figure 7). In all of the situations, a non-homogeneous area can be noticed in the lower right corners of the mosaics, which were caused by shadows of clouds during the image acquisition.

The importance of the radiometric block adjustment can be verified by analysing Figure 7(a). This figure presents the mosaics without corrections, and the influence of the BRDF and the illumination variation are visible. However, when the BRDF correction 
was applied (Figure $7(b)$ ), a variation of the illumination still occurred, which is seen as the higher intensity level of the southern flight line.

When using the irradiance data from the camera's sensor, a higher variation of luminosity can be observed (Figure $7(\mathrm{c})$ ). Analysing the UAV attitude data, it was found that the radiance sensor was leaning towards the Sun during the first flight strip, which caused higher irradiance values as confirmed in Figure 4. In addition, the roll and pitch attitude parameters varied more in the first than in the second flight strip. Considering this, the initial coefficient factors for the radiometric bundle adjustment were affected, as was the final mosaic.

For the mosaic with the BRDF_rela_ground correction (Figure $7(d)$ ), the differences in the mosaic were probably caused by differences in the illumination conditions at the ground station and in the area where the images were acquired; the spectroradiometer was located in western part of the survey area, approximately $800 \mathrm{~m}$ away from the end of the first flight strip. The BRDF_rela correction (Figure $7(\mathrm{e})$ ) provided the best uniformity when visually assessed.

\subsection{Uniformity of the mosaics}

Figure 8 shows the CVs for each band. The factors ranged from 0.08 to 0.11 for the mosaic without corrections (no_corr). Considering the BRDF_rela correction, which presented the lowest RMSE, the CV was $0.07-0.10$, which is half the variation of the BRDF_rela_cam coefficients (0.15-0.19). Additionally, for the BRDF_only and BRDF_rela_ground corrections, the CVs were $0.07-0.11$ and $0.08-0.10$, respectively. In previous studies of agricultural areas, CVs of approximately 0.06 were obtained in agricultural fields where the correction was successful (Honkavaara et al. 2013). The factor is dependent not only on the homogeneity of the mosaic but also on the object characteristics. In this area, the forested part increased the CVs. The CVs were higher in the spectral bands centred on 506.07 and $650.28 \mathrm{~nm}$, probably due to the spectral position of these bands, which were located along the borders of each sensor of the camera. These bands were left out from the detailed analysis. The results of analyses of the CVs were consistent with the visual assessment of the mosaics in Section 3.2.

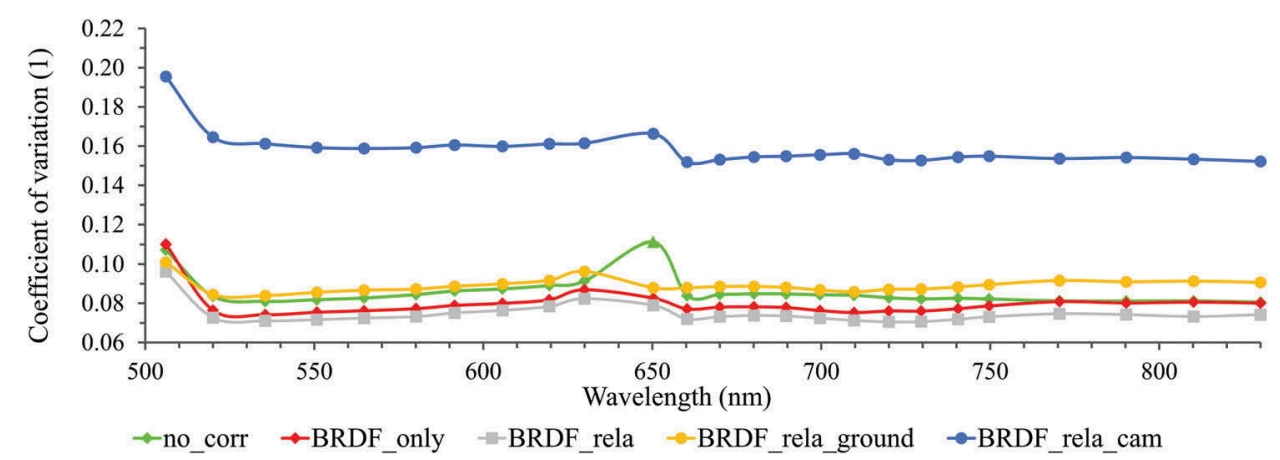

Figure 8. Coefficients of variation of the radiometric tie points for each type of correction (no_corr, BRDF_only, BRDF_rela_ground, BRDF_rela_cam and BRDF_rela). 


\subsection{Quality of the spectral data}

Figure 9 shows the spectral response of the targets acquired in the field that were considered to be ground truth as well as those acquired in the image mosaics that were generated with the different imaging processing parameters. The BRDF_rela_cam correction was not used in Figure 9, since it presented higher discrepancies and would affect the data visualization.

The spectra measured on the ground were different from the UAV mosaics. The reference spectra were smooth, whereas the spectra measured from the UAV mosaics were more variable. These differences could be explained by the vibrations of the platform and by sensor calibration inaccuracies; in the laboratory measurements, we did not observed such variations, and thus, the impacts of vibrations are a more likely explanation.

Substantially large differences also appear between the UAV mosaic-based spectra and the ground reference spectra for many targets. Many factors may have caused these differences. When considering the image corrections, depending on the case, the correction parameters used were of different quality. One should also notice that the BRDF correction was determined for the vegetation and thus would not be the correct model for non-vegetated targets and would deteriorate the results for those targets. The different measurement geometries of the ground spectrometer and the FPI camera may also cause differences in the spectral values (Schaepman-Strub et al. 2006). These aspects are considered in the following for each target by analysing the RMSEs between the spectra from the mosaics and the reference spectra.

Considering target 1 (black EVA), the lowest RMSE values were obtained for the BRDF_rela_ground and BRDF_rela corrections, which were 0.02 and 0.06 , respectively. The graphical analysis of these spectra in Figure 9 shows that the higher differences occurred in the near infrared region. This can be explained by the target location in the field, which was adjacent to sugarcane, which has a high reflectance in this part of the electromagnetic spectrum and increases the amount of diffuse light, thereby affecting the observed reflectance.

For the grey targets (targets 2 and 11), the lowest RMSE values varied between 0.070.08 and $0.04-0.06$ for the no_corr and BRDF_rela corrections, respectively. The BRDF_rela correction thus presented the best results for this target. The spectra from the mosaic with the BRDF_rela_ground correction were biased and showed clearly lower reflectance values than the reference spectra. A possible explanation for this bias is the use of irradiance values acquired in the field from a fixed position for the initial values of the relative correction, which did not correspond to the irradiance in the object during data acquisition, as well as an inaccurate BRDF correction.

For the white targets (targets 5, 8, 9, and 10), the lowest RMSE values varied between 0.02-0.08 and 0.03-0.04 for the BRDF_only and BRDF_rela corrections, respectively. Similar to the grey targets, biases were observed in the spectra with the BRDF_rela_ground correction.

For the healthy sugarcane leaf spectra (target 3), the best RMSE values were obtained with the BRDF_rela (0.09) and BRDF_only (0.10) corrections. For the diseased sugarcane leaf (target 4), the best RMSE values were observed with the BRDF_rela_ground (0.04) and BRDF_only (0.08) corrections. When analysing these differences, it is necessary to 
(a)

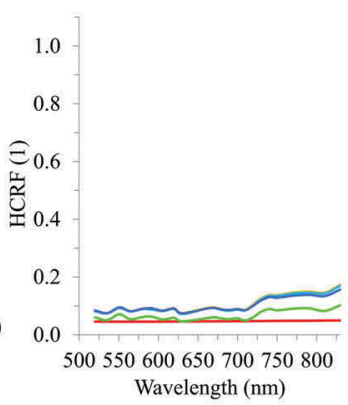

(d)

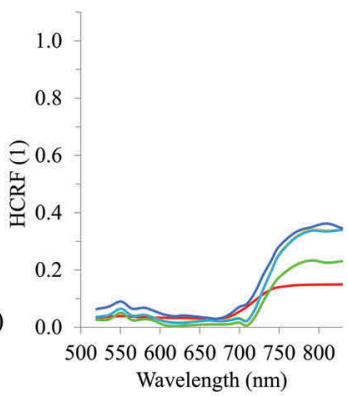

(g)

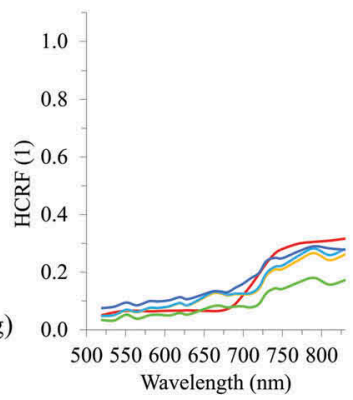

(b)
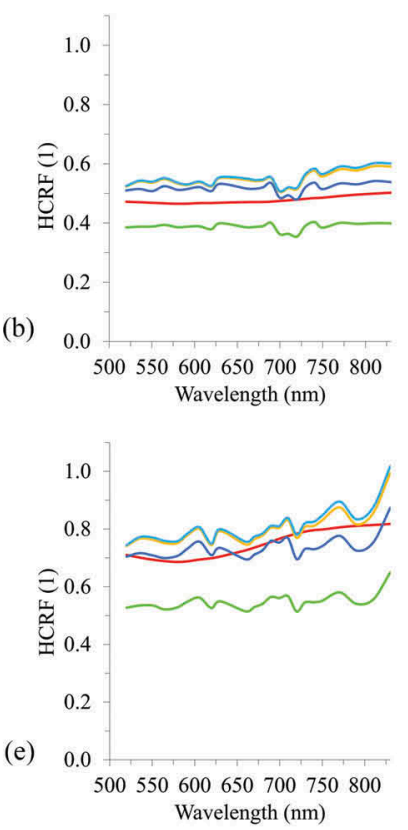

(f)
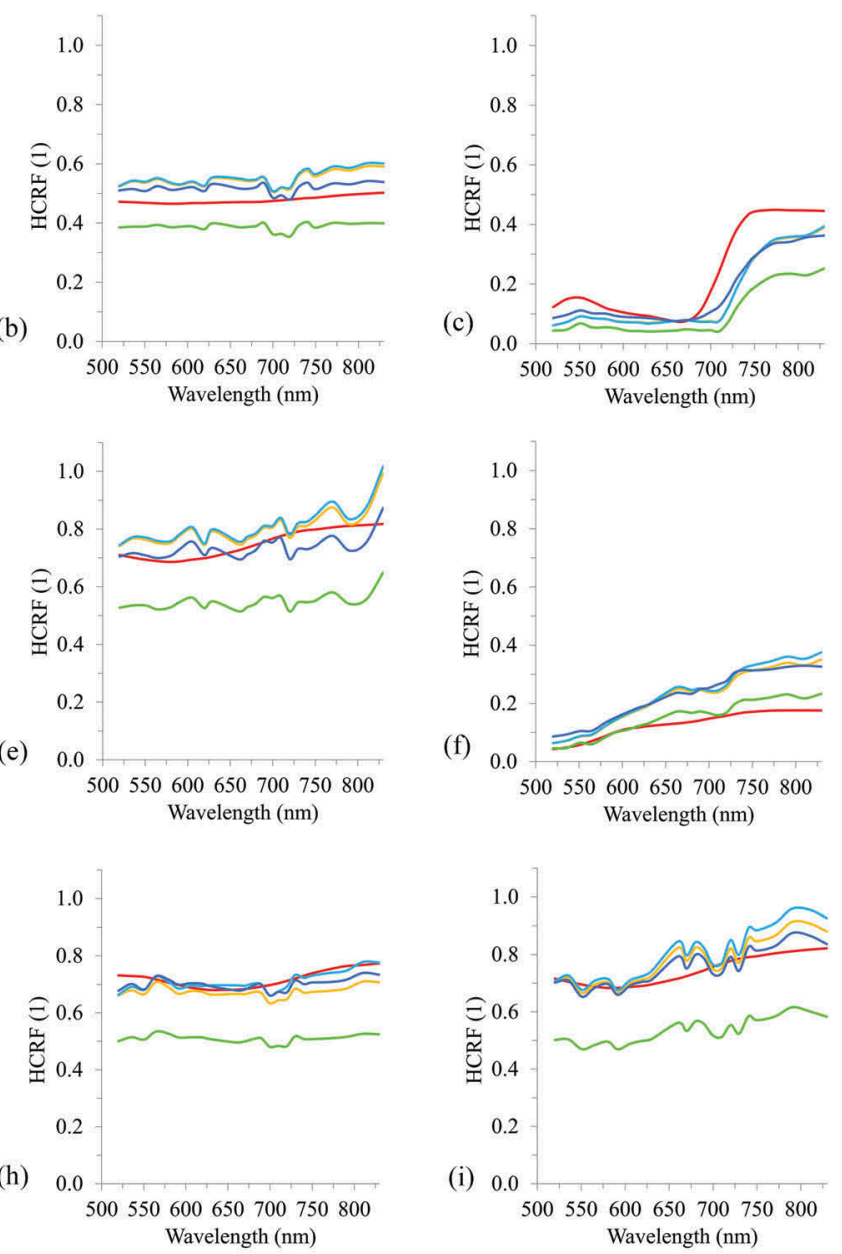

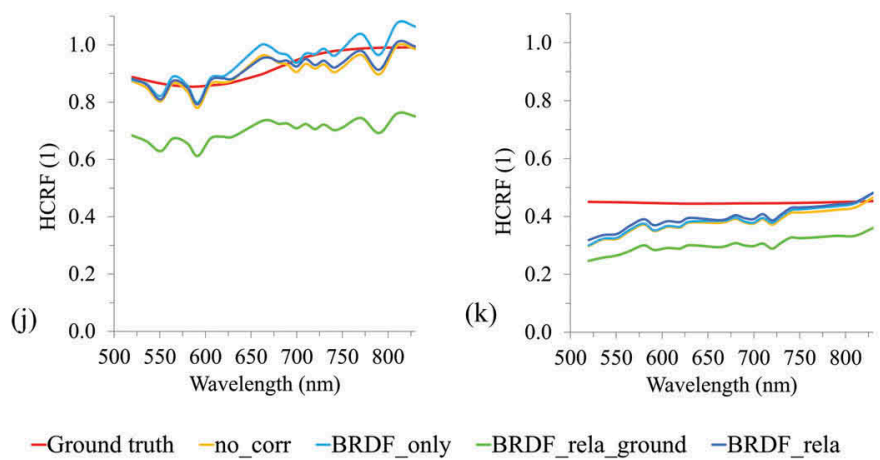

Figure 9. HCRF spectra acquired in the field, considered to be the ground truth, and HDRF spectra acquired for the no_corr, BRDF_rela, BRDF_only, and BRDF_rela_ground corrections for the targets: (a) 1 - black EVA; (b) 2 - grey EVA; (c) 3 - healthy sugarcane; (d) 4 - diseased sugarcane; (e) 5 white EVA; (f) 6 - sandy soil; (g) 7 - weed; (h) 8 - white EVA; (i) 9 - white EVA; (j) 10 - white EVA; and (k) 11 - grey EVA. 
consider the different geometries of the field- and image-based measurements. In the field reference measurement, the HCRF spectra were acquired for leaves in the case of UAV imaging. In the mosaics, the HDRF values were measured for the canopy and were thus influenced by the different stages of development of the vegetation, the background, and, most importantly, the sugarcane structure and image acquisition geometry, both of which caused the influence of vegetation and shadows in the spectra measured from the images.

The RMSEs were lower for the weeds (target 7) than for the sugarcane. This could be explained by the fact that the reference spectra, which were acquired with the ASD spectroradiometer in the field, were collected for the canopy, which is more similar to the measurement geometry from the images. The best RMSE values were 0.04 for the BRDF_rela and no_corr corrections. Analysing the spectra of this target in Figure 9, it can be noticed that the higher differences occur in the red-edge and near-infrared regions.

Finally, for the soil (target 6), the BRDF_rela_ground and no_corr corrections gave the lowest RMSE values of 0.03 and 0.10 , respectively. However, the BRDF_rela correction showed RMSE values that were more similar to the no_corr correction, 0.11 . Many factors may have impacted the large RMSE values, including effects from the adjacent sugarcane and issues with the BRDF corrections.

Table 3 presents the mean differences and RMSEs for the eleven reference targets for each correction method. Similar values for the mean differences for the imaging processing no_corr (-0.012), BRDF_only (-0.023), and BRDF_rela $(-0.012)$ corrections, which were different from those of BRDF_rela_ground (0.111) and BRDF_rela_cam (0.829), were noticed. Even with equal mean differences between the no_corr and the BRDF_rela corrections, the latter showed a better accuracy with a RMSE of $11.35 \%$. Moreover, for the BRDF_rela_cam correction, the RMSE (275.27\%) was more than twenty times higher than the RMSE found for the mosaic produced by corrections based on the radiometric block adjustment using the same initial parameters (BRDF_rela).

\subsection{Comparison and outlook}

The empirical results showed that the BRDF_rela correction provided the best results with the test data set with the Brazilian vegetation (Figure $7(d)$ ). Similar results were obtained by Hakala et al. (2013) in agricultural fields with wheat and barley. The results showed that the camera radiance sensor, as it was implemented in the UAV, should not be used as a source of information for radiometric processing without corrections. A compensation for the attitude variations from the irradiance observations might be conducted in future works. Another possibility is to utilize the ground irradiance, but only after positioning it closer to the centre of the survey area. In this study, better results were obtained using the initial relative correction factors calculated without the use of irradiance measures after applying the BRDF correction.

Table 3. Mean differences and RMSE values for each correction.

\begin{tabular}{lccccc}
\hline & \multicolumn{5}{c}{ Correction } \\
\cline { 2 - 6 } Statistics & no_corr & BRDF_only & BRDF_rela_cam & BRDF_rela_ground & BRDF_rela \\
\hline Mean differences (1) & $-0.012 \pm 0.057$ & $-0.023 \pm 0.056$ & $0.829 \pm 0.864$ & $0.111 \pm 0.095$ & $-0.012 \pm 0.049$ \\
RMSE (\%) & 13.17 & 13.69 & 275.27 & 33.77 & 11.35 \\
\hline
\end{tabular}


Considering the right side of the mosaic, where the images were under partial cloud cover, it can be noticed that the images could not be corrected with the current version of the radiometric block adjustment. It is recommended to avoid using this part of the mosaic for remote-sensing applications. In future studies, the performance of shadow correction algorithms (Adeline et al. 2013) or the separation of shadowed and nonshadowed areas in the analysis should be evaluated.

The importance of the BRDF effects and their correction can also be seen in Liesenberg, Galvão, and Ponzoni (2007), which presented differences in the classification of physiognomies for different seasonalities and geometries of view using data from the Multi-angle Imaging SpectroRadiometer. The results of Burkart et al. (2015) showed differences in three vegetation indices by considering different geometries of view in a wheat study site. Lastly, Aasen (2016) published results about uncorrected BRDF effects and showed different spectral responses and different normalized difference vegetation index values for a barley field. These results highlight the importance of the correction of BRDF effects.

\section{Conclusion}

The objective of this investigation was to optimize a hyperspectral UAV-based remotesensing system to the Brazilian environment. The sensor was a novel, tunable FPI-based frame format hyperspectral imager. The images were acquired and processed using five different parameters.

An empirical study was carried out in an area composed of sugarcane and forest vegetation. The spectral settings of 25 bands in the range from 500 to $900 \mathrm{~nm}$ were selected based on the spectral responses of vegetation objects of interest in the research area. Hyperspectral UAV imagery was captured under varying illumination conditions, which are typical to the area.

Different options for correcting the image radiometric differences due to illumination changes were studied. The methods employed in this study included the use of spectral image data directly without any corrections, with the utilization of data from ground and from UAV-based irradiance sensors, and with image-based corrections in the context of a radiometric block adjustment of a multiview image block. Independent reference targets were used as quality control information for each approach to determine which approach best fits the Brazilian environment.

In general, the methodology considering the irradiance measures acquired by the spectroradiometer located at a fixed position in the field, such as the one collected by the irradiance sensor of the camera that was mounted in the UAV, did not produce the best results. The best results were obtained by considering only the image information and applying the radiometric block adjustment.

The accurate spectral data are of interest for many applications. Hyperspectral UAV remote sensing presents excellent opportunities for various environmental applications, but it also sets new challenges for radiometric processing because UAVs are often operated in varying illumination conditions. The irradiance observations from onboard the UAV or from the ground are expected to be helpful for correcting the illumination changes, but further improvements to the hardware are required in order to obtain reliable irradiance data. An important challenge was the effects of the BRDF 
due to object anisotropy. The radiometric block adjustment-based method was used for the first time in the Brazilian environment, and it was shown to efficiently compensate for both illumination variations and BRDF effects. Further improvements will improve the accuracy, versatility, and reliability of the approach.

\section{Acknowledgements}

The authors would like to acknowledge the support of the São Paulo Research Foundation for the Brazilian-Finnish joint project "Unmanned Airborne Vehicle Based 4D Remote Sensing for Mapping Rain Forest Biodiversity and its Change in Brazil" (FAPESP - Grant Number 2013/ 50426-4; Academy of Finland - decision number 273806), as well the research provided by the Conselho Nacional de Desenvolvimento Científico e Tecnológico (CNPq) through a master's degree scholarship: [Grant Number 130871/2014-1].

\section{Disclosure statement}

No potential conflict of interest was reported by the authors.

\section{Funding}

This work was supported by the Academy of Finland: [Grant Number 273806], Conselho Nacional de Desenvolvimento Científico e Tecnológico: [Grant Number 130871/2014-1], Fundação de Amparo à Pesquisa do Estado de São Paulo: [Grant Number 2013/50426-4].

\section{References}

Aasen, H., A. Burkart, A. Bolten, and G. Bareth. 2015. "Generating 3D Hyperspectral Information with Lightweight UAV Snapshot Cameras for Vegetation Monitoring: From Camera Calibration to Quality Assurance." ISPRS Journal of Photogrammetry and Remote Sensing 108: 245-259. doi:10.1016/j.isprsjprs.2015.08.002.

Aasen, H. 2016. "Influence of the Viewing Geometry Within Hyperspectral Images Retrieved from UAV Snapshot Cameras." International Archives of the Photogrammetry, Remote Sensing and Spatial Information Sciences 257-261. doi:10.5194/isprs-annals-III-7-257-2016.

Adeline, K. R. M., M. Chen, X. Briottet, S. K. Pang, and N. Paparoditis. 2013. "Shadow Detection in Very High Spatial Resolution Aerial Images: A Comparative Study." ISPRS Journal of Photogrammetry and Remote Sensing 80: 21-38. doi:10.1016/j.isprsjprs.2013.02.003.

Berveglieri, A., and A. M. G. Tommaselli 2016. "Exterior Orientation of Hyperspectral Frame Images Collected with UAV for Forest Applications." In Proc. Int. Arch. Photogrammetry, Remote Sens. Spatial Inf. Sci., Eur. Calibration and Orientation Workshop, 45-50. doi:10.5194/isprsarchives-XL-3W4-45-2016

Berveglieri, A., A. M. G. Tommaselli, N. N. Imai, E. A. W. Ribeiro, R. B. Guimarães, and H. Honkavaara. 2016. "Identification of Successional Stages and Cover Changes of Tropical Forest Based on Digital Surface Model Analysis." IEEE Journal of Selected Topics in Applied Earth Observations and Remote Sensing 9 (12): 5385-5397. doi:10.1109/JSTARS.2016.2606320.

Burkart, A., H. Aasen, L. Alonso, G. Menz, G. Bareth, and U. Rascher. 2015. "Angular Dependency of Hyperspectral Measurements over Wheat Characterized by a Novel UAV Based Goniometer." Remote Sensing 7 (1): 725-746. doi:10.3390/rs70100725.

Büttner, A., and H. P. Röser. 2014. "Hyperspectral Remote Sensing with the UAS 'Stuttgarter Adler'System Setup, Calibration and First Results." Photogrammetrie-Fernerkundung-Geoinformation 2014 (4): 265-274. doi:10.1127/1432-8364/2014/0217. 
Colomina, I., and P. Molina. 2014. "Unmanned Aerial Systems for Photogrammetry and Remote Sensing: A Review." ISPRS Journal of Photogrammetry and Remote Sensing 92: 79-97. doi:10.1016/j.isprsjprs.2014.02.013.

Everaerts, J. 2009. "NEWPLATFORMS-Unconventional Platforms (Unmanned Aircraft Systems) for Remote Sensing." Official Publication, no. 56.

Hakala, T., E. Honkavaara, H. Saari, J. Mäkynen, J. Kaivosoja, L. Pesonen, and I. Pölönen. 2013. "Spectral Imaging from UAVs under Varying Illumination Conditions." International Archives of the Photogrammetry, Remote Sensing and Spatial Information Sciences 189-194. doi:10.5194/ isprsarchives-XL-1-W2-189-2013.

Honkavaara, E., R. Arbiol, L. Markelin, L. Martinez, M. Cramer, S. Bovet, L. Chandelier, et al. 2009. "Digital Airborne photogrammetry-A New Tool for Quantitative Remote sensing?-A State-Ofthe-Art Review on Radiometric Aspects of Digital Photogrammetric Images." Remote Sensing 1 (3): 577-605. doi:10.3390/rs1030577.

Honkavaara, E., T. Hakala, L. Markelin, T. Rosnell, H. Saari, and J. Mäkynen. 2012a. "A Process for Radiometric Correction of UAV Image Blocks." Photogrammetrie-Fernerkundung-Geoinformation 2012 (2): 115-127. doi:10.1127/1432-8364/2012/0106.

Honkavaara, E., J. Kaivosoja, J. Mäkynen, I. Pellikka, L. Pesonen, H. Saari, H. Salo, T. Hakala, L. Marklelin, and T. Rosnell. 2012b. "Hyperspectral Reflectance Signatures and Point Clouds for Precision Agriculture by Light Weight UAV Imaging System." ISPRS Annals Photogramm Remote Sens Spatial Information Sciences I-7: 353-358. doi:10.5194/isprsannals-l-7-353-2012.

Honkavaara, E., H. Saari, J. Kaivosoja, I. Pölönen, T. Hakala, P. Litkey, J. Mäkynen, and L. Pesonen. 2013. "Processing and Assessment of Spectrometric, Stereoscopic Imagery Collected Using a Lightweight UAV Spectral Camera for Precision Agriculture." Remote Sensing 5 (10): 5006-5039. doi:10.3390/rs5105006.

Hruska, R., J. Mitchell, M. Anderson, and N. F. Glenn. 2012. "Radiometric and Geometric Analysis of Hyperspectral Imagery Acquired from an Unmanned Aerial Vehicle." Remote Sensing 4 (9): 27362752. doi: $10.3390 /$ rs4092736.

Laliberte, A. S., M. A. Goforth, C. M. Steele, and A. Rango. 2011. "Multispectral Remote Sensing from Unmanned Aircraft: Image Processing Workflows and Applications for Rangeland Environments." Remote Sensing 3 (11): 2529-2551. doi:10.3390/rs3112529.

Liesenberg, V., L. S. Galvão, and F. J. Ponzoni. 2007. "Variations in Reflectance with Seasonality and Viewing Geometry: Implications for Classification of Brazilian Savanna Physiognomies with MISR/Terra Data." Remote Sensing of Environment 107 (1): 276-286. doi:10.1016/j. rse.2006.03.018.

Lucieer, A., Z. Malenovský, T. Veness, and L. Wallace. 2014. "HyperUAS-Imaging Spectroscopy from a Multirotor Unmanned Aircraft System." Journal of Field Robotics 31 (4): 571-590. doi:10.1002/rob.21508.

Mäkynen, J., C. Holmlund, H. Saari, K. Ojala, and T. Antila. 2011. “Unmanned Aerial Vehicle (UAV) Operated Megapixel Spectral Camera." Proceedings of SPIE, Electro-Optical Remote Sensing, Photonic Technologies, and Applications 81860Y-1. doi:10.1117/12.897712.

McCoy, R. M. 2005. Field Methods in Remote Sensing. New York, NY: Guilford Press.

Milton, E. J., M. E. Schaepman, K. Anderson, M. Kneubühler, and N. Fox. 2009. "Progress in Field Spectroscopy." Remote Sensing of Environment 113: S92-S109. doi:10.1016/j.rse.2007.08.001.

Näsi, R., E. Honkavaara, P. Lyytikäinen-Saarenmaa, M. Blomqvist, P. Litkey, T. Hakala, N. Viljanen, T. Kantola, T. Tanhuanpää, and M. Holopainen. 2015. "Using UAV-Based Photogrammetry and Hyperspectral Imaging for Mapping Bark Beetle Damage at Tree-Level." Remote Sensing 7 (11): 15467-15493. doi:10.3390/rs71115467.

Oliveira, R. A., A. M. Tommaselli, and E. Honkavaara. 2016. "Geometric Calibration of a Hyperspectral Frame Camera." The Photogrammetric Record 31 (155): 325-347. doi:10.1111/phor.12153.

Rees, W. G. 2013. Physical Principles of Remote Sensing. Cambridge, UK: Cambridge University Press. Richter, R., and D. Schläpfer. 2002. "Geo-Atmospheric Processing of Airborne Imaging Spectrometry Data. Part 2: Atmospheric/Topographic Correction." International Journal of Remote Sensing 23 (13): 2631-2649. doi:10.1080/01431160110115834. 
Saari, H., I. Pölönen, H. Salo, E. Honkavaara, T. Hakala, C. Holmlund, J. Mäkynen, R. Mannila, T. Antila, and A. Akujärvi. 2013. "Miniaturized Hyperspectral Imager Calibration and UAV Flight Campaigns." SPIE Remote Sensing. 888910-888910-12. International Society for Optics and Photonics. doi:10.1117/12.2028972

Schaepman-Strub, G., M. E. Schaepman, T. H. Painter, S. Dangel, and J. V. Martonchik. 2006. "Reflectance Quantities in Optical Remote sensing-Definitions and Case Studies." Remote Sensing of Environment 103 (1): 27-42. doi:10.1016/j.rse.2006.03.002.

Senop. 2017. "Datasheet VIS-NIR Snapshot Hyperspectral Camera for UAVs". Snapshot hyperspectral camera. Accessed 23 June 2017. http://view.24mags.com/mera/datasheet-hyperspectralcamera

Smith, G. M., and E. J. Milton. 1999. "The Use of the Empirical Line Method to Calibrate Remotely Sensed Data to Reflectance." International Journal of Remote Sensing 20 (13): 2653-2662. doi:10.1080/014311699211994.

Stein, A., F. D. van der Meer, and B. Gorte. 2006. Spatial Statistics for Remote Sensing. Vol. 1. New York, NY: Springer Science \& Business Media.

Suomalainen, J., N. Anders, S. Iqbal, G. Roerink, J. Franke, P. Wenting, D. Hünniger, H. Bartholomeus, R. Becker, and L. Kooistra. 2014. "A Lightweight Hyperspectral Mapping System and Photogrammetric Processing Chain for Unmanned Aerial Vehicles." Remote Sensing 6 (11): 11013-11030. doi:10.3390/rs61111013.

Tommaselli, A. M., R. A. Oliveira, L. Y. Nagai, N. N. Imai, G. T. Miyoshi, E. Honkavaara, and T. Hakala. 2015. "Assessment of Bands Coregistration of a Light-Weight Spectral Frame Camera for UAV." GeoUAV - ISPRS Geospatial Week.

Tsai, F., and W. Philpot. 1998. "Derivative Analysis of Hyperspectral Data." Remote Sensing of Environment 66 (1): 41-51. doi:10.1016/S0034-4257(98)00032-7.

Walthall, C. L., J. M. Norman, J. M. Welles, G. Campbell, and B. L. Blad. 1985. "Simple Equation to Approximate the Bidirectional Reflectance from Vegetative Canopies and Bare Soil Surfaces." Applied Optics 24 (3): 383-387. doi:10.1364/AO.24.000383.

Zarco-Tejada, P. J., V. González-Dugo, and J. A. Berni. 2012. "Fluorescence, Temperature and Narrow-Band Indices Acquired from a UAV Platform for Water Stress Detection Using a MicroHyperspectral Imager and a Thermal Camera." Remote Sensing of Environment 117: 322-337. doi:10.1016/j.rse.2011.10.007. 